\title{
Establishment and optimization of fluid pipe network models based on topological analysis algorithm
}

\author{
Peng Cheng ${ }^{1,2 *}$, Jinhua Zhang ${ }^{2}$, Dan Bai ${ }^{1}$ \\ ${ }^{1}$ Xi'an University of Technology, Xi'an, Shanxi Province 710048, China \\ ${ }^{2}$ College of Mathematics and Statistics, North China University of Water Resources and Electric Power, Zhengzhou 450045, \\ China
}

Corresponding Author Email: ncwucheng@163.com

https://doi.org/10.18280/ijht.360430

Received: 23 February 2018

Accepted: 13 June 2018

\section{Keywords:}

topological analysis, fluid pipe network, two pipe networks, optimization

\begin{abstract}
Recent years has seen the proliferation of various fluid pipe networks in our living and working environments. With the growth in size, complexity and scale, fluid pipe networks are faced with numerous problems like pipe collision and bursting. To prevent these problems, ensure the operation efficiency and save cost, this paper probes into the structure and performance of two fluid pipe networks that backs up each other. Based on topological analysis algorithm, the two fluid pipe networks were converted into topological graphs, and created topological models involving pipe elements and nodes. Through optimization of the models, the author proposed the two-pipe connection mode for the two pipe networks. The optimization strategy was then verified through a simulation application in a tree-shaped water injection system. The results show that our strategy can effectively optimize the two-pipe connection, ensure the operation efficiency and save the network cost. The research findings provide a good reference to the design and optimization of fluid pipe networks in China.
\end{abstract}

\section{INTRODUCTION}

The continuous improvement of living standards has increased the presence of various fluid pipe networks in our living and working environments, such as the pipe networks for domestic water supply, heat supply, rainwater drainage, wastewater drainage, fire water supply, industrial water supply and oilfield water injection [1]. To satisfy the needs of life and work, all types of pipe networks are continuously growing in size and complexity. Many problems (e.g. the collision, wrong connection and connection failure between pipes) will emerge if the fluid pipe networks are constructed empirically without rational analysis or design [2]. The ensuing to resource waste, cost hike and network instability will make it impossible for the fluid pipe networks to fulfill the living and working demands. As a result, in-depth analysis of the structure and performance of fluid pipe networks is needed to rationalize and optimize the design of these networks.

In general, a fluid pipe network consists of three parts: source power, nodes and connecting pipes. These parts interact with each other, forming a complex yet complete system [3]. The research focus of fluid pipe networks lies in reducing cost and enhancing efficiency through structural optimization. For instance, Afshar, and Ribas et al., optimized the pipe layout of water supply pipe network by the genetic algorithm [4-5]. Sinha and Pandey, and Carrión et al., put forward reliability evaluation methods after examining the structural performance of different fluid pipe networks [6-7]. Chang established an equivalent model of fluid pipe network [8].

In addition, some scholars have introduced topological analysis, which focuses on the spatial correlation of each element in a set, to explore fluid pipe networks. For example, Zhang et al., (2017) relied on topological analysis algorithm to optimize the fluid pipe network layout through the optimization of the node positions and connections. Popular topological algorithms include Prime algorithm and genetic algorithm [9].

To sum up, the existing studies mainly deal with the optimization or reliability evaluation of a single fluid pipe network, failing to explore the optimal connection between two or more fluid pipe networks. In fact, the connection between multiple fluid pipe networks is a common issue, due to the continuous expansion of these networks. Therefore, this paper attempts to optimize the connection between two fluid pipe networks, aiming to save cost and enhance operation efficiency, in addition to optimizing the layout of fluid pipe networks.

To optimize the connection between two fluid pipe networks, this paper converts fluid pipe networks into topological graphs based on the topology analysis algorithm, and sets up the topology models of the fluid pipe networks. After optimizing these models, the author proposed a method to optimize the connection between the two fluid pipe networks. The proposed method was proved feasible through the simulation application to an actual water injection system. The research findings provide a good reference for the design and optimization of fluid pipe networks in China.

\section{MODEL CONSTRUCTION}

This paper considers two fluid pipe networks (hereinafter referred to as the system) that back up each other, that is, the primary pipe network and the secondary pipe network serve as the backup network for each other through the optimal connection mode. This connection pattern ensures the 
operation efficiency (e.g. preventing failure-induced outage), lowers the investment and operation costs, and optimizes the network layout.

\subsection{Establishment of fluid pipe element model}

The first step of model construction is to analyze the topology of the fluid pipe networks. For simplicity, the fluid is assumed to flow at a constant speed, and each fluid pipe network is considered as the combination of multiple pipe elements via nodal connections. In addition, all pipe elements are assumed to obey the law of conservation of energy [10]. Let me be the node on one side and $\mathrm{n}$ be the node on the other side of pipe element $i$. If the elevation of node $m$ is greater than that of node $n$, then the fluid will flow from $m$ ton, and the pressure at node $m$ will be greater than that of node $n$. The energy resulted from elevation difference can be expressed as:

$p_{i}^{m}-\left(\rho \cdot g \cdot \Delta P_{i}\right)+\left(\rho \cdot g \cdot\left(H_{i}^{m}-H_{i}^{n}\right)\right)=P_{i}^{n}$

where $P_{i}^{m}$ and $P_{i}^{n}$ are the pressures at node $\mathrm{m}$ and node $\mathrm{n}$, respectively; $\rho$ is the fluid density; $g$ is the acceleration of gravity; $H_{i}^{m}$ and $H_{i}^{n}$ are the elevations of node $\mathrm{m}$ and node $\mathrm{n}$, respectively; $\Delta P_{i}$ is the pressure loss of pipe element $i$. The value of $\Delta P_{i}$ can be calculated by the Darcy's law [11]:

$\Delta P_{i}=\lambda \cdot \frac{l_{i} \cdot v_{i}^{2}}{2 \cdot g \cdot d_{i}}=\lambda \cdot \frac{8 \cdot l_{i} \cdot Q_{i}^{2}}{\pi \cdot g \cdot d_{i}^{5}}$

where $l_{i}, v_{i}, d_{i}$ and $Q_{i}$ are the length, fluid flow speed, inner diameter and flow of pipe element $\mathrm{i} ; \lambda$ is the head loss coefficient.

The flow in each pipe element changes with the pressure loss between the nodes on both sides of the element. The three variation conditions are explained below:

(a) If $\Delta p_{i} \geq p^{r e q}$, then

$$
Q_{i}=\left\{\begin{array}{l}
\text { satisfies the } q^{\text {req }} \\
\text { partly satisfies } 0<q<q^{r e q} \\
0(\text { no flow })
\end{array}\right.
$$

(b) If $p^{\min _{i}{ }^{\text {req }}}$, then

$0<Q_{i}<q^{r e q}$

(c) If $\Delta p_{i}<p^{\text {min }}$, then $Q_{i}=0$ (no flow).

where $q^{r e q}$ is the required flow of the node of the pipe element; $p^{r e q}$ is the minimum pressure under the required flow; $p^{m i n}$ is the minimum pressure for the fluid to reach the node.

\subsection{Establishment of fluid node model}

It is assumed that the flow through each node is constant, i.e. the incoming flow equals the outgoing flow at the node. In this case, the flow of node $\mathrm{m}$ is made up of the incoming flow $I_{m}$, the flow $Q_{i}$ of the pipe element $\mathrm{i}$ connected to this node and the flow loss $q_{m}$ of this node:

$$
I_{m}=Q_{i}+\sum_{n \in S_{m}} q_{m n}, \quad m=0,1, \cdots, M-1
$$

where $S_{m}$ is the set of serial numbers of the nodes connected to node $\mathrm{m} ; \mathrm{M}$ is the total number of nodes in the primary pipe network.

\subsection{Calculation of model cost}

The overall cost is positively correlated with the cost per pipe element, node and connection. Hence, the cost objective function can be established as:

$$
C=\min \left(\sum_{m=0}^{M-1} \sum_{n=0}^{N-1} \gamma_{m n} \cdot \omega_{m n} \cdot l_{m n}\right)
$$

where $C$ is the cost of the system; $\gamma_{m n}$ is the connection coefficient between node $\mathrm{m}$ in the primary pipe network and node $\mathrm{n}$ in the secondary pipe network $\left(\gamma_{m n}=1\right.$ if the two nodes are connected; $\gamma_{m n}=0$ if otherwise); $\omega_{m n}$ is the unit cost of connecting the two pipe elements; $M$ and $N$ are the total number of nodes in the primary and secondary pipe networks, respectively; $l_{m n}$ is the connection length. The value of $l_{m n}$ can be calculated as:

$$
l_{m n}=\sqrt{\left(x_{m}-x_{n}\right)^{2}+\left(y_{m}-y_{n}\right)^{2}+\left(z_{m}-z_{n}\right)^{2}}
$$

where $\left(x_{m}, y_{m}, z_{m}\right)$ are the coordinates of node $\mathrm{m}$ in the primary pipe network; $\left(x_{n}, y_{n}, z_{n}\right)$ the coordinates of node $\mathrm{n}$ in the secondary pipe network. In actual pipe networks, the connections between the nodes are not necessarily linear. To improve the model accuracy, additional nodes can be designed at the break points when the pipe networks are converted into topological graphs.

\section{MODEL OPTIMIZATION}

The fluid pipe networks were converted into topological graphs, taking the energy supply stations and intermediate stations as nodes and the pipes as edges. The topological models of the primary and secondary pipe networks were then optimized by single parent genetic algorithm, in reference to relevant literature [12]. The connection between the two pipe networks was optimized by the flow in Figure 1.

After setting up the topologies, the parameters of each node, namely, pressure $P$ and flow $Q$, were calculated from equations (1) (4). Then, the importance of each pipe element was evaluated against the availability of nodes, flow or pipe elements (the availability is defined as the ratio of the number of nodes, the flow or the number of pipe elements after troubleshooting to the total number of nodes, the total flow or the total number of pipe elements when the system works normally). The satisfactory nodes determined in importance evaluation were sorted. Then, the nodes of the primary and secondary pipe networks were allocated to sets $S 1$ and $S 2$, respectively. 


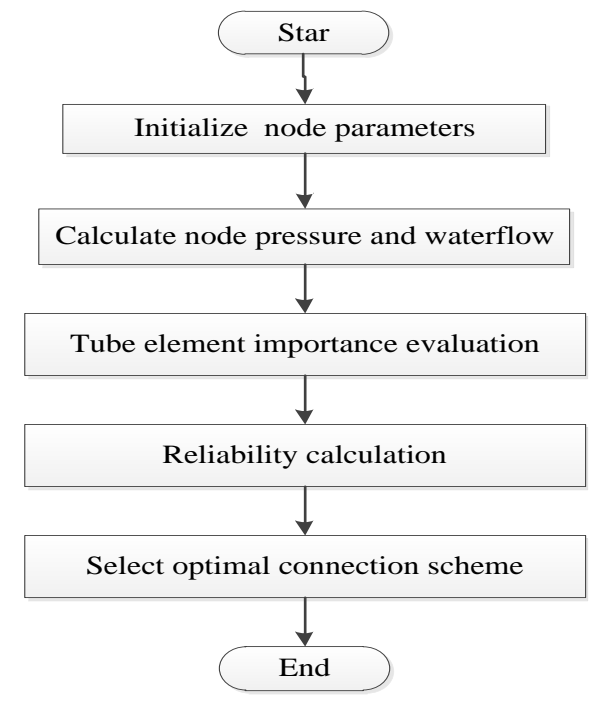

Figure 1. Optimization flow of the connection between the two pipe networks

The connection mode was optimized as follows. First, one node (to simulate single-pipe connection) or two nodes (to simulate two-pipe connection) were selected randomly from the two sets. Under no external pressure, the author judged whether the pressure of a node could satisfy the pressure required for the other node. If the result was positive, the reliability of each connection was calculated and the cost was computed by equation (5). Then, the connection mode with higher reliability and lower cost was identified. Under external pressure, the reliability and cost were computed directly for each connection. Then, the connection mode with higher reliability and lower cost was identified.

Inspired by the previous research [13], the mean reliability $R_{m}$ of each node is calculated and taken as the reliability of that node:

$R_{m}=\frac{\sum_{D=1}^{M A X} \sum_{t=1}^{24} Q_{m t}}{\sum_{D=1}^{M A X} \sum_{t=1}^{24} Q_{m t}^{r e q}}$

where $Q_{m t}$ is the flow of node i within the t-th hour; $D$ is the number of sampling days; $Q_{m t}^{r e q}$ is the flow required for node $\mathrm{m}$ within the $\mathrm{t}$-th hour. If $R_{m}=0$, then node $\mathrm{m}$ has no flow in that period, which requires inspection and repair to eliminate the failure; If $0<R_{m}<1$, then node $\mathrm{m}$ works imperfectly (e.g. lack of flow supply and possible pipe damage), which also requires inspection; If $R_{m}=1$, then node $\mathrm{m}$ works normally.

Referring to the previous research [13], the instantaneous reliability $R S_{j}$ of the system can be computed as:

$$
R S_{j}=\frac{\sum_{m=0}^{M-1} Q_{m j}^{r e q}\left(Q_{m j} / Q_{m j}^{r e q}\right)}{\sum_{m=0}^{M-1} Q_{m j}^{r e q}}
$$

where $Q_{m j}$ is the flow of node m at the j-th hour; $Q_{m j}^{r e q}$ is the flow required for node $\mathrm{m}$ at the $\mathrm{j}$-th hour. If $R S_{j}=0$, then the system stopped running; If $0<R S_{j}<1$, then node m works imperfectly (e.g. lack of flow supply and possible pipe damage); If $R S_{j}=1$, the system works normally.

\section{SIMULATION APPLICATION}

The established model optimization method was applied to a typical tree-shaped water injection system in Qinggang County, northeastern China's Heilongiiang Province. To begin with, the primary and secondary pipe networks of the system were separately converted into topological structures, and the relevant parameters were calculated. Figures 2 and 3 present the topological graphs optimized by the genetic algorithm.

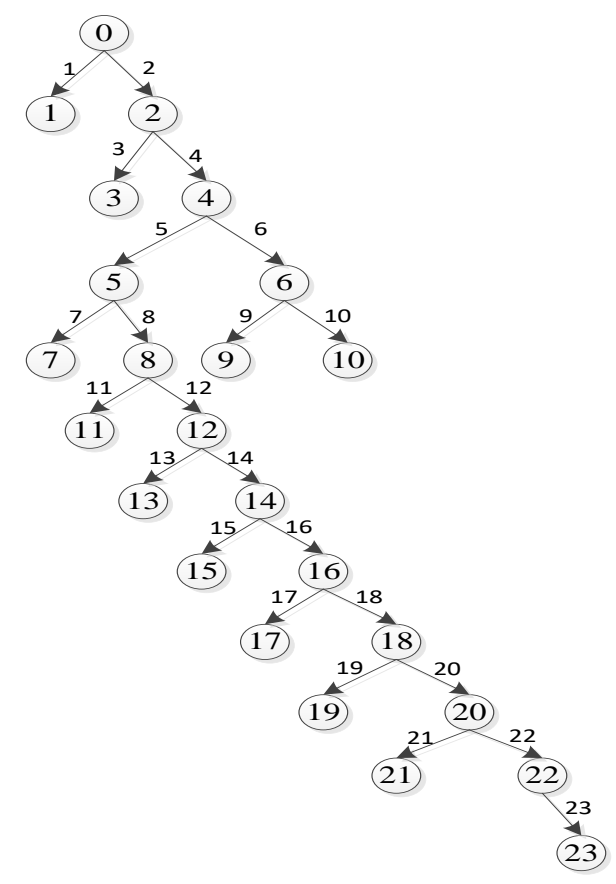

Figure 2. Topological graph of the primary fluid pipe network

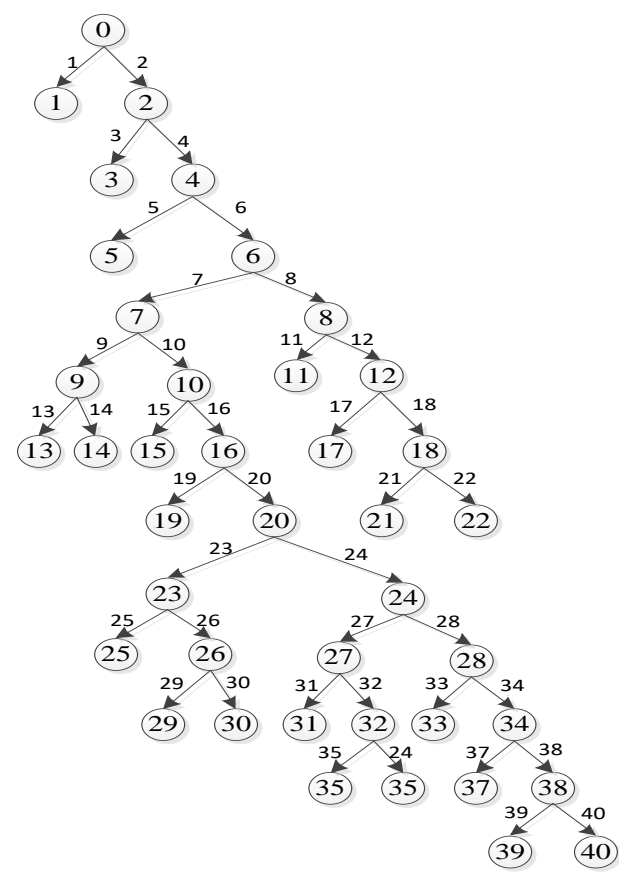

Figure 3. Topological graph of the secondary fluid pipe network 


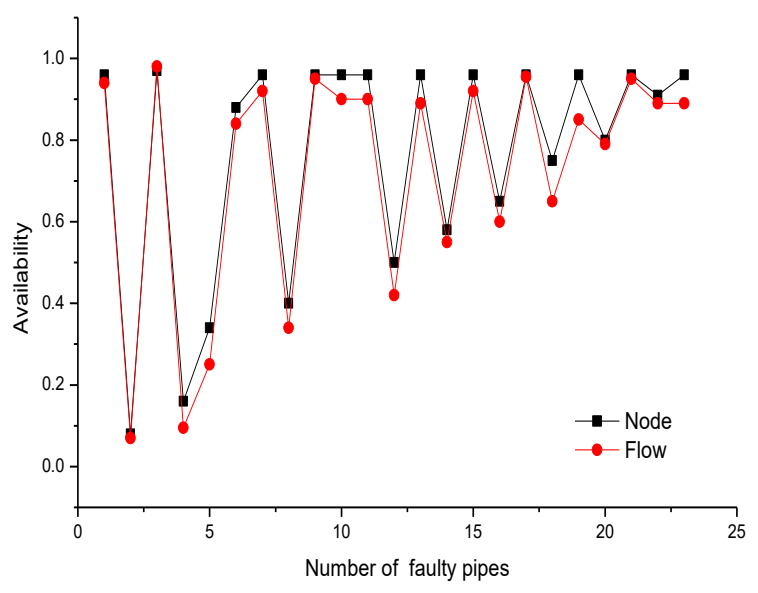

Figure 4. Node and flow availabilities of the primary fluid pipe network under pipe element failure

To optimize the connection between the primary and secondary networks, the importance of each pipe element in the two pipe networks was evaluated separately, and the node and flow availabilities were calculated under the failure of each pipe element. The results are shown in Figures 4 and 5.

The importance of a pipe element is negatively correlated with the availabilities of the corresponding node and flow when the pipe element fails. Here, the availability of 0.5 is treated as the critical value, and the pipe elements with smaller-than- 0.5 node and flow availabilities are considered as relatively important. As shown in Figures 4 and 5, the pipe elements 2\#,
$4 \#, 5 \#, 8 \#$ and $12 \#$ in the primary pipe network were relatively important, so were the pipe elements $2 \#, 4 \#, 6 \#, 7 \#$ and $10 \#$ in the secondary pipe network. Thus, the serial numbers of such pipe elements in the primary pipe network were allocated to set $S 1$, while those in the secondary pipe network to set $S 2$ : $S 1=\{2,4,5,8,12\}$ and $S 2=\{2,4,6,7,10\}$.

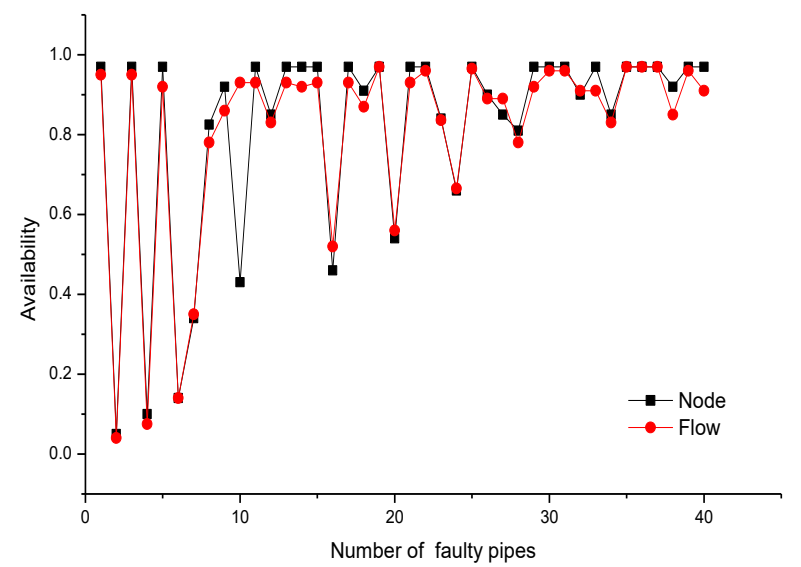

Figure 5. Node and flow availabilities of the secondary fluid pipe network under pipe element failure

Two nodes were randomly selected from the two sets to link up the two networks, and the connected pipe elements were numbered. Two-pipe connection, more reliable than one-pipe connection, was adopted. The node connections are recorded in Table 1 below.

Table 1. Node connections between the primary and secondary pipe networks

\begin{tabular}{c|c|c|c|c|c|c|c|c|c|c}
\hline Number & 1 & 2 & 3 & 4 & 5 & 6 & 7 & 8 & 9 & 10 \\
\hline \multirow{2}{*}{ Node number } & $2-2$ & $2-2$ & $2-4$ & $2-4$ & $2-6$ & $2-6$ & $2-7$ & $2-7$ & $2-10$ & $2-10$ \\
& $5-4$ & $12-4$ & $5-2$ & $8-2$ & $5-2$ & $8-2$ & $4-10$ & $5-2$ & $4-7$ & $5-2$ \\
\hline Number & 11 & 12 & 13 & 14 & 15 & 16 & 17 & 18 & 19 & 20 \\
\hline \multirow{2}{*}{ Node number } & $4-2$ & $4-2$ & $4-4$ & $4-4$ & $4-6$ & $4-6$ & $4-7$ & $4-7$ & $4-10$ & $4-10$ \\
& $5-4$ & $8-4$ & $5-2$ & $8-2$ & $5-2$ & $8-2$ & $5-2$ & $8-2$ & $5-2$ & $8-2$ \\
\hline Number & 21 & 22 & 23 & 24 & 25 & 26 & 27 & 28 & 29 & 30 \\
\hline \multirow{2}{*}{ Node number } & $5-2$ & $5-2$ & $5-4$ & $5-4$ & $5-6$ & $5-6$ & $5-7$ & $5-7$ & $5-10$ & $5-10$ \\
& $8-6$ & $12-4$ & $8-6$ & $12-2$ & $8-2$ & $8-4$ & $8-2$ & $8-6$ & $8-6$ & $8-6$ \\
\hline Number & 31 & 32 & 33 & 34 & 35 & 36 & 37 & 38 & 39 & 40 \\
\hline \multirow{2}{*}{ Node number } & $8-2$ & $8-2$ & $8-4$ & $8-4$ & $8-6$ & $8-6$ & $8-7$ & $8-7$ & $8-10$ & $8-10$ \\
& $12-4$ & $12-6$ & $12-2$ & $12-6$ & $12-2$ & $12-10$ & $12-2$ & $12-6$ & $12-2$ & $12-6$ \\
\hline
\end{tabular}

After the connection, the reliability of each network and the cost of the connections were calculated. The results are displayed in Figures 6 8.

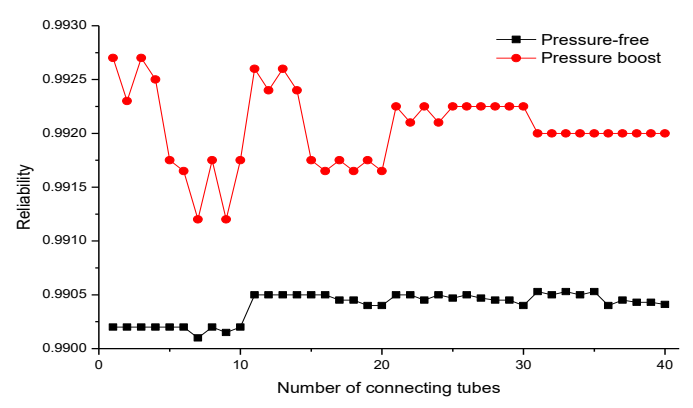

Figure 6. Reliability of the primary network after the connection

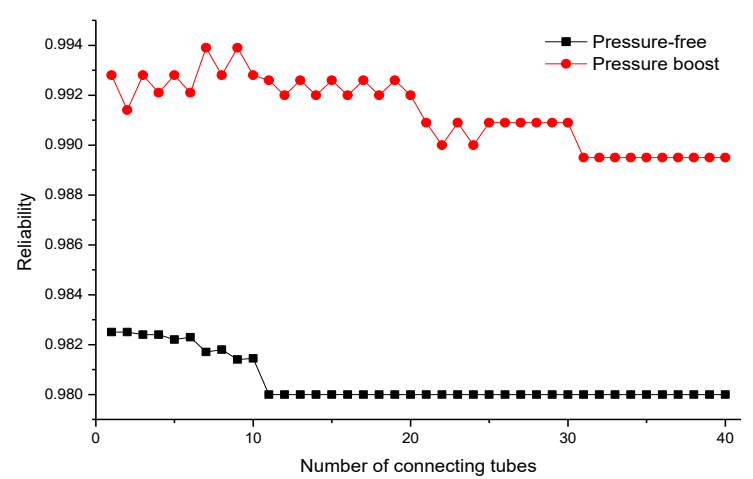

Figure 7. Reliability of the secondary network after the connection 


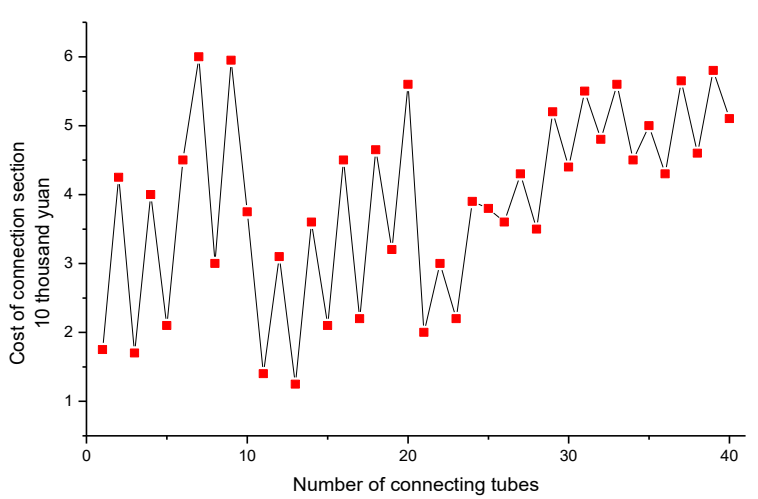

Figure 8. Cost of the connections between the two networks

It can be seen from the above figures that the two networks were more reliable under external pressure than without external pressure. This is attributable to the following facts: without external pressure, some nodes have no flow after connection due to the pressure limit; with external pressure, the flow between the nodes is not limited by pressure; the connecting pipe elements with high reliability tend to have high cost. Considering both reliability and cost, it is recommended to select the connecting pipe elements with high reliability and low cost. Through comparison between reliabilities and costs, it is learned that pipe element $13 \#$ had the lowest cost while pipe element $1 \#$ boasted the highest reliability. In general, the two pipe elements enjoyed high reliability at a low cost. Thus, the optimal connection mode is: connecting nodes $2 \#$ and $5 \#$ of the primary network to nodes $2 \#$ and $4 \#$ of the secondary network, respectively; connecting nodes $2 \#$ and $5 \#$ of the primary network to nodes $4 \#$ and $2 \#$ of the secondary network, respectively.

\section{CONCLUSIONS}

This paper establishes fluid pipe network models based on topological analysis. The established models were proved to have high computing accuracy, considering the pressure loss and the elevation difference of nodes on the two sides of each pipe. On this basis, the author identified the optimal connection between two fluid pipe networks that back up each other, through importance evaluation, reliability calculation and cost computation. Then, the proposed connection optimization strategy was verified through a simulation application in a tree-shaped water injection system. The simulation results show that our method is rational and feasible in that it simultaneously ensures the operation efficiency and reduces the cost of the fluid pipe networks. The research findings provide a good reference to the design and optimization of fluid pipe networks.

\section{ACKNOWLEDGMENTS}

Key Scientific and Technological Research Projects in Henan Province (no: 162102110130); study on Identification of solute transport parameters in unsaturated soils.

\section{REFERENCES}

[1] Sui J, Yang L, Hu Y. (2016). Complex fluid network optimization and control integrative design based on nonlinear dynamic model tr. Chaos Solitons \& Fractals the Interdisciplinary Journal of Nonlinear Science \& Nonequilibrium \& Complex Phenomena 89: 20-26. http://doi.org/10.1016/j.chaos.2015.09.009

[2] Ayad A, Awad H, Yassin A. (2013). Developed hydraulic simulation model for water pipeline networks. Alexandria Engineering Journal 52(1): 43-49. http://doi.org/10.1016/j.aej.2012.11.005

[3] Almazyad AS, Seddiq YM, Alotaibi AM, Alnasheri AY, Bensaleh MS, Obeid AM. (2014). A proposed scalable design and simulation of wireless sensor network-based long-distance water pipeline leakage monitoring system. Sensors $14(2)$ : 3557-3577. http://doi.org/10.3390/s140203557

[4] Afshar MH. (2012). Rebirthing genetic algorithm for storm sewer network design. Scientia Iranica 19(1): 1119. http://doi.org/10.1016/j.scient.2011.12.005

[5] Ribas PC, Yamamoto L, Polli HL, Arruda LVR, NevesJr F. (2013). A micro-genetic algorithm for multiobjective scheduling of a real-world pipeline network. Engineering Applications of Artificial Intelligence 26(1): 302-313. http://doi.org/10.1016/j.engappai.2012.09.020

[6] Sinha SK, Pandey MD. (2010). Probabilistic neural network for reliability assessment of oil and gas pipelines. Computer-Aided Civil and Infrastructure Engineering 17(5): 320-329. http://doi.org/10.1111/1467-8667.00279

[7] Carrión A, Solano H, Gamiz ML, Debón A. (2010). Evaluation of the reliability of a water supply network from right-censored and left-truncated break data. Water Resources Management 24(12): 2917-2935. http://doi.org/10.1007/s11269-010-9587-y

[8] Chang YL. (2001). A study on model simplification technology and calculation method of water flooding pipeline network system. Acta Petrolei Sinica 22(2): 95100.

[9] Zhang H, Liang Y, Zhou X, Yan X, Qian C, Liao Q. (2017). Sensitivity analysis and optimal operation control for large-scale waterflooding pipeline network of oilfield. Journal of Petroleum Science \& Engineering 154. http://doi.org/1016/j.petrol.2017.04.019

[10] Tang CY, Chen HX, Zhu B. (2010). Research on modeling of water supply pipeline network for meeting energy demands and energy conservation. Process Automation Instrumentation 31(3): 12-15.

[11] Valiantzas JD. (2008). Explicit power formula for the darcy-weisbach pipe flow equation: application in optimal pipeline design. Journal of Irrigation \& Drainage Engineering 134(4): 454-461. http://doi.org/10.1061/(asce)0733-9437(2008)134:4(454)

[12] Bakri B, Arai Y, Inakazu T, Koizumi A, Yoda H, Pallu S. (2015). Selection and concentration of pipeline mains for rehabilitation and expansion of water distribution network is. Procedia Environmental Sciences 28: 732742. http://doi.org/10.1016/j.proenv.2015.07.086

[13] Tanyimboh TT, Tabesh M, Burrows R. (2001). Appraisal of source head methods for calculating reliability of water distribution networks. Journal of Water Resources Planning \& Management 127(4): 206-213. http://doi.org/10.1061/(asce)0733-9496(2001)127:4(206) 